\title{
Le livre de raison de Guillaume Tabourot et Jeanne Bernard, notables bourguignons (Heures à l'usage de Rome, Université McGill, MS 154)
}

ARIANE BERGERON-FOOTE

Archiviste-paléographe (École des chartes)

Ouvrages précieux pour leurs propriétaires successifs, les livres d'Heures sont parfois riches en renseignements sur les premières familles qui les ont conservés. Certains livres d'Heures sont ainsi augmentés d'un livre de raison, offrant mémoires, évènements marquants et chronologie des temps forts d'une famille, consignés sur les pages de garde ou dans les marges laissées blanches. Peint en Bourgogne, le livre d'Heures ms. McGill 154 (v. 14801490) contient un livre de raison du couple Guillaume Tabourot et Jeanne Bernard (née en 1582), couvrant les années 1606 à 1645. Guillaume Tabourot (1573-1644) était le fils du célèbre littérateur Étienne Tabourot, seigneur des Accords. Cette contribution propose une transcription commentée du livre de raison Tabourot-Bernard. Le texte autographe consigné par Guillaume Tabourot reflète bien la montée et réussite sociale des Tabourot au sein de la haute société bourguignonne, où ils s'investirent dans les affaires parlementaires et municipales, mais aussi s'engagèrent dans des projets artistiques et architecturaux.

Books of Hours are precious objects for their successive owners, for they are often rich in information concerning the earliest families who kept them. Certain Books of Hours are thus augmented by a livre de raison, supplying recollections, noteworthy occasions, and a chronology of the major events in a family's history, which have been recorded on flyleaves or in the page margins. Painted in Burgundy, the Book of Hours McGill, MS 154 (v. 1480-1490) contains a livre de raison of the couple Guillaume Tabourot and Jeanne Bernard (born in 1582), covering the years 1606 to 1645. Guillaume Tabourot (1573-1644) was the son of the famous poet and author Etienne Tabourot, Seigneur des Accords. This contribution offers a transcription with commentary of Tabourot-Bernard's livre de raison. The autographed text recorded by Guillaume Tabourot clearly reflects the rise and social success of the Tabourots within Burgundian high society, being involved in parliamentary and municipal affairs as well as artistic and architectural projects.

$\mathrm{I}^{1}$ est des documents qui sont particulièrement émouvants. Les livres de raison en font partie, parce qu'ils témoignent de la vie quotidienne et des temps forts de l'existence humaine, en toute simplicité, qu'il s'agisse d'une famille connue ou non. Lorsque cette famille a eu une importance culturelle et sociale, comme celle des Tabourot, le document n'en est que plus intéressant 
encore. La famille Tabourot, implantée en Bourgogne, en Franche-Comté et en Champagne-Ardenne, participa au rayonnement de la culture bourguignonne aux $\mathrm{XV}^{\mathrm{e}}$ et $\mathrm{XVI}^{\mathrm{e}}$ siècles à travers les arts et les honneurs.

Le livre d'Heures, conservé à Montréal au Département des Livres rares et collections spécialisées de l'Université McGill sous la cote MS 154, renferme en toute fin de volume (fol. $126 \mathrm{v}^{\circ}-127 \mathrm{v}^{\circ}$ ) un livre de raison manuscrit où sont consignés les naissances et décès de membres de la famille de Guillaume II Tabourot (1573-1644) et de Jeanne Bernard (née en 1582). Ce livre de raison, dont nous proposons dans ce qui suit une édition, couvre les années 1606 à $1645^{1}$. Le couple Tabourot-Bernard a eu une progéniture nombreuse de onze enfants qui sont répertoriés au fur et à mesure de leur naissance. On y décline aussi quelques-uns des temps forts ou des trépas marquants pour la famille, comme celle de la mort de la mère de Guillaume Tabourot, Gabrielle Chiquot de Montpastey (morte en 1628), femme d'Étienne Tabourot, puissant ligueur, homme de lettres bourguignon et auteur, entre autres, des célèbres Bigarrures du seigneur des Accords (Paris, éditions successives 1572, 1583, 1585)². Aussi,

1. Le livre de raison fut signalé par Brenda Dunn-Lardeau dans son article, «De quelques livres d'Heures remarquables conservés dans les collections montréalaises » in Livros de Horas. Imaginário da devoção privada : manuscritos, éd. Delmira Espada Custódio et Maria Adelaïde Miranda (Lisbonne : Biblioteca Nacional de Portugal, Babel, 2016 version numérique, version imprimée sous presse), 245-264. Nicole Reynaud («Un peintre français de la fin du XVe siècle : le Maitre des Prélats bourguignons », in Études d'art français offertes à Charles Sterling [Paris : Presses Universitaires de France, 1975], 151-163) connaissait l'existence du livre d'Heures par les catalogues successifs de vente aux enchères avant son entrée dans les collections de l'Université McGill (voir Dunn-Lardeau, 34). Nous remercions le Département des livres rares et collections spécialisées de l'Université McGill, la Beinecke Rare Book and Manuscript Library de l'Université Yale ainsi que le possesseur du dessin à l'encre de J.-A. De Baïf par G. Tabourot de leur aimable autorisation de reproduction des illustrations. Enfin, cet article s'inscrit dans le projet de recherche sur les livres d'Heures conservés au Québec financé par le CRSH (2014-2018) sous la direction de Brenda Dunn-Lardeau.

2. Étienne Tabourot (1547-1590) est né près de Dijon à Saint-Apollinaire (situé au nord-est de Dijon, département de la Côte-d'Or), terre dont son père Guillaume était le seigneur (il est dit seigneur de la Tour de Saint-Apollinaire). Il est l'aîné des quatre enfants de Bernarde Thierry et Guillaume Tabourot. Son oncle Jean Tabourot (1520-1595), chanoine à Langres, publiera en 1589, sous l'anagramme « Thoinot Arbeau ", son Orchésographie, premier traité connu d'art chorégraphique. Guillaume Ir Tabourot, père d'Étienne (et donc grand-père du Guillaume II Tabourot dont nous transcrivons le livre de raison), maître extraordinaire de la chambre des comptes, mourut en 1561 (J. d'Arbaumont, Armorial de la chambre des comptes de Dijon [Dijon : Lamarche, 1881], 152). Étienne Tabourot, pour sa part, décède en septembre ou octobre 1590 d'une affection du foie : il repose à la cathédrale Saint-Bénigne de Dijon. Sur 
soigneusement consigné, ce livre de raison enregistre le décès d'Étienne Bernard (mort en 1609), père de Jeanne Bernard, beau-père de Guillaume Tabourot depuis leur mariage en 1606. Notons que la mort d'Étienne Tabourot (1549-1590), père de Guillaume, n'est pas évoquée, pour la simple raison que Guillaume Tabourot fait débuter son livre de raison en 1606 et donc bien après le trépas d'Étienne Tabourot, mort relativement jeune quand son fils aîné Guillaume n’avait que dix-sept ans. Étienne Tabourot ou « Tabourot des Accords ", dit le «Rabelais bourguignon » n'en était pas moins une figure centrale pour cette famille ${ }^{3}$. Une brève analyse quantitative fait apparaître que ce livre de raison consigne huit décès pour douze naissances. Autres temps forts pour la famille Tabourot-Bernard, deux mariages $(1606,1630)$, un voyage en Italie (1606), une entrée royale, celle de Louis XIII (1629) et deux prises d'habits $(1630,1631)$.

\section{Ex-libris et devise " A tous accords» : des Heures peintes en Bourgogne}

Outre le livre de raison, nous savons que ce manuscrit a figuré un temps dans la bibliothèque d'un membre de la famille Tabourot grâce à l'ex-libris qui figure sur le premier feuillet du calendrier. On y lit leur devise «A tous accords » et

Étienne Tabourot, voir Georges Choptrayanovitch, Étienne Tabourot des Accords (1549-1590). Étude sur sa vie et son cuvre littéraire (Dijon : Faculté des lettres, 1935 et Genève : Slatkine reprints, 1970). Voir aussi l'édition la plus récente : Estienne Tabourot, Les Bigarrures du Seigneur des Accords, éd. Francis Goyet, 2 vols. (Genève : Droz, 1986).

3. Étienne Tabourot donna en 1587 une nouvelle édition du Dictionnaire des rimes françoises : Premierement composé par Jean Le Fevre Dijonnais, Chanoine de Langres et de Bar-sur-Aube; et depuis augmenté, corrigé et mis en bon ordre, par le Seigneur des Accords (Paris : Jean Richer, 1587). Étienne Tabourot peut se prévaloir d'un réseau bien ancré auprès des notables de Dijon et de Langres. Il met en tête de cette nouvelle édition des vers français, latins, italiens et grecs dus à un groupe de Dijonnais et Langrois qui bien qu'ecclésiastiques, jurisconsultes, clercs, étaient aussi poètes amateurs. Parmi ceux-ci, on retrouve les personnages suivants : Bénigne Des Barres, Jacques Tabourot, Jean Tabourot, Alexandre Tabourot, Guillaume et Pierre Tabourot (Étienne Tabourot publie les vers latins de ses fils, alors âgés respectivement de 12 et 9 ans ; le premier, Guillaume, auteur du présent livre de raison : «1584. Guliel. Taborotius, aetat. 12 an. » ; le second, Pierre : «1584. Petrus Taborotius aet. 9 an. » (Dictionnaire des rimes françoises, 1587, sig. e iii verso), Guillaume et Jean Berbisey, Guillaume Le Goulx, Bernard Coussin, F. Juret. Toutes ces familles se retrouvent dans le livre de raison de Guillaume Tabourot, comme époux, parrains et marraines. 


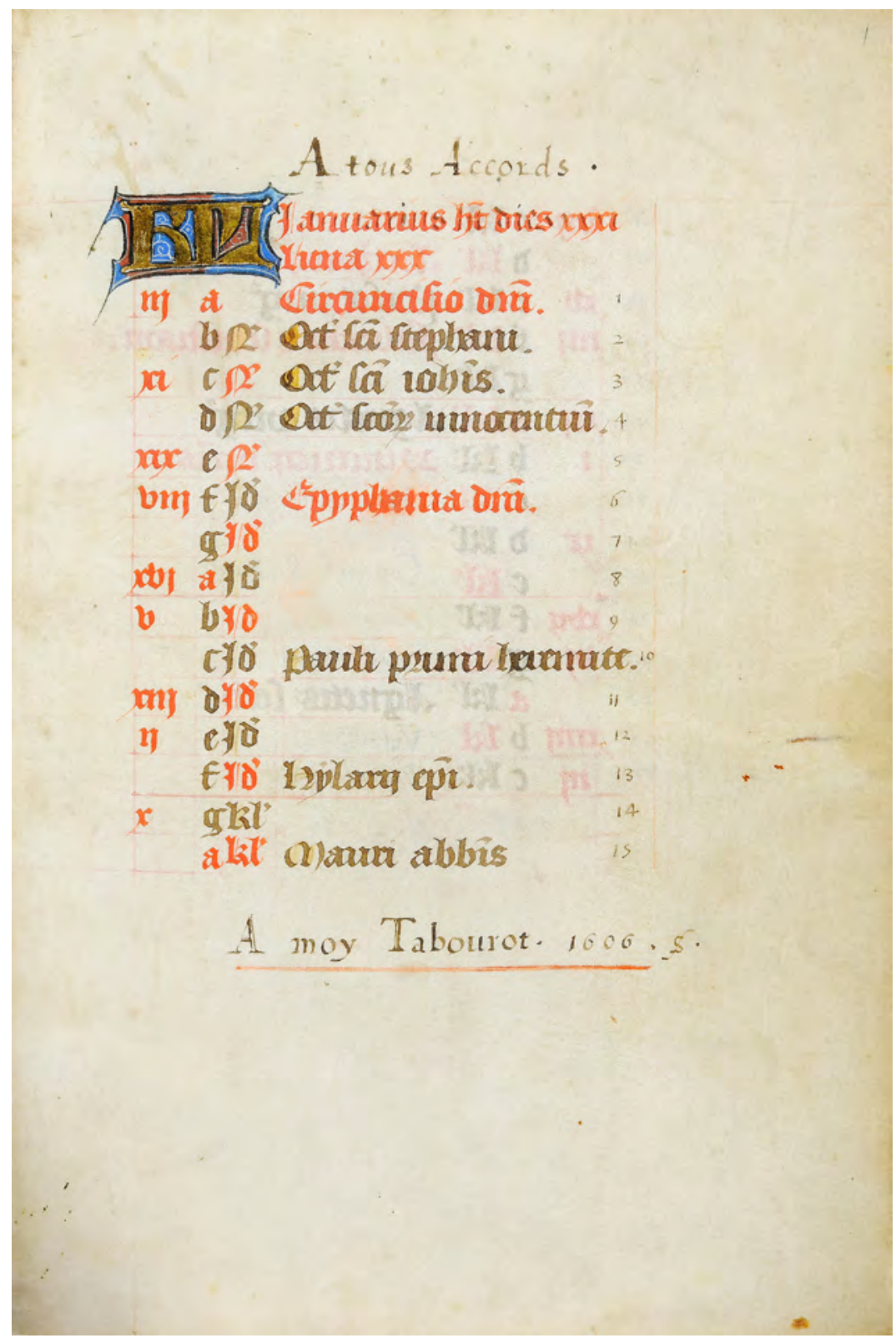

Fig. 1. Livre d'Heures, Calendrier, mois de janvier, ex-libris et devise de Guillaume Tabourot. Montréal, Université McGill, LRCS, MS 154. 
l'ex-libris de Guillaume Tabourot : «A moy Tabourot. 1606 » (fig. 1)4. La date de 1606 qui figure dans la marge inférieure du premier feuillet est suivie d'une «fermesse » (ou S fermé), signe employé dans les décors aux XVI et XVII ${ }^{e}$ siècles, symbole entre autres d'affection, d'amour et de fidélité ${ }^{5}$. Parmi les autres marques de personnalisation, signalons la devise "A tous accords », répétée au feuillet 57 dans un phylactère soutenu par un ange, mais rajoutée a posteriori ${ }^{6}$. Le livre d'Heures conservé à McGill (MS 154) est une production bourguignonne locale : si son usage est celui universel de Rome, avec un calendrier clairsemé qui ne trahit que quelques saints honorés en Bourgogne, les enluminures qu’il

4. À noter que - outre la devise - le manuscrit ne comporte pas d'éléments héraldiques liés à la provenance Tabourot. La famille anoblie portait les armoiries suivantes : «d'azur au chevron d'or, accompagné de trois tambours d'or, alias d'argent, au chef aussi d'argent, chargé d'un lion courant (ou passant) de sable ". Voir Lachesnaye-Desbois, Dictionnaire de la noblesse, $3^{\mathrm{e}}$ édition (Paris : Schlesinger, 1873), tome XVIII, colonne 727. Dans le seul écu comportant des éléments héraldiques (on relèvera un écu resté vide dans la bordure inférieure du feuillet 65, tracé en camaïeu d’or), peint dans la bordure inférieure de l'Annonciation (fol. 17), on reconnait un homme nu et tonsuré, portant un écu d'azur chargé d'une lettre $\mathrm{C}$ et d'un instrument qui pourrait être un compas (ouvert en chevron). Ces armoiries demeurent pour l'heure non identifiées. Toutefois, notons qu'il existait à Dijon une famille « Le Compasseur », conseillers au Parlement de Bourgogne : Claude Le Compasseur fut reçu en 1620 conseiller clerc. Les armoiries de la famille Le Compasseur se blasonnent : d'azur à trois compas ouverts en chevron d'or (Pierre Palliot, Le parlement de Bourgongne, son origine, son établissement et son progrès [Dijon : Paillot, 1649], 289-290). Nous remercions ici M. Marc Smith, professeur à l'École des chartes, pour la confirmation du meuble «compas » et l'association possible - mais pour l'heure non confirmée - avec une famille «Le Compasseur ». Ce manuscrit a-t-il appartenu au XV siècle à une autre famille, puis été acquis - par exemple à l'occasion d'un mariage - ou hérité par la famille Bernard ou Tabourot?

5. Un ouvrage ayant appartenu à Guillaume Tabourot présente un ex-libris semblable, copié de la même main. Il s'agit d’un exemplaire de Diogène Laërce (Venise : G. B. Bertoni, 1606), doté sur la page de titre de l'inscription " Taboroti est. 1607 » et d'une seconde mention « A tous accords ", suivi de la même fermesse. Ce livre, provenant d'une collection privée, est recensé par Gilles Banderier, « Ex bibliotheca Taborotiana », in Annales de Bourgogne, 73 (2001) : 437-440; les deux pages de titre, avec les inscriptions à rapprocher de celles du livre d'Heures de McGill, sont reproduites aux pages 438-439.

6. Sur les marques de personnalisation de ces Heures liées à la famille Tabourot, voir Dunn-Lardeau, 36. Sur la devise "A tous accords" adoptée par les Tabourot, voir Choptrayanovitch, 31-32. Étienne Tabourot employa l'appellation « seigneur des accords " pour la première fois dans ses Bigarrures. L'auteur nous dit qu'il a " des accords en sa seigneurie pour contenter les humeurs diverses des plus rébarbatifs et joyeux " (Avis au lecteur, édition de 1582). Pierre Perrenet parle effectivement de " cette seigneurie bizarre que l'on chercherait en vain dans un dictionnaire topographique de la région » (Pierre Perrenet, Estienne Tabourot, sa famille et son temps [Dijon : Éditions du raisin, 1926], 1). 
renferme sont clairement attribuables, pour certaines d'entre elles, au Maître des Prélats bourguignons, un artiste français actif en Bourgogne pendant le dernier quart du XV $\mathrm{XV}^{\mathrm{e}}$ siècle ${ }^{7}$. Le manuscrit peut être daté d'environ 1480-1490 et a dû appartenir à deux, voire trois, propriétaires précédant le couple TabourotBernard. En 1606, mariés depuis peu, les Tabourot-Bernard ont donc choisi un manuscrit peint en Bourgogne pour y consigner les temps forts de cette famille résolument bourguignonne. Le Maître des Prélats bourguignons est connu pour avoir fourni des livres d'Heures aux familles d'influence dijonnaises et bourguignonnes, tels les Berbisey (cette famille est citée dans le livre de raison de Guillaume Tabourot et Jeanne Bernard en 1622, au feuillet 127 : Marthe Berbisey est la marraine de Marc Antoine Tabourot) ${ }^{8}$. Une des mains de Guillaume Tabourot (celle qui signe et date «A moy Tabourot. 1606 » dans la marge inférieure du premier feuillet du calendrier, la même qui rajoute la devise de la famille «A tous accords » dans la marge supérieure du calendrier) est à notre avis la même main qui rajoute au calendrier clairsemé des noms de saints (voir par exemple aux feuillets $1 \mathrm{r}^{\mathrm{o}}, 1 \mathrm{v}^{\mathrm{o}}$, et passim) $)^{9}$, personnalisant un

7. Nous renvoyons aux travaux de Nicole Reynaud, voir Reynaud, « Un peintre français... », 151-163; et Nicole Reynaud, "La Bourgogne ", in Les manuscrits à peintures en France, 1440-1520 (Paris : Flammarion, 1993), 393 et notices $\mathrm{n}^{\text {os }} 222-226$, pages $394-398$. Voir aussi, plus récemment, les travaux de Brenda Dunn-Lardeau qui évoque les Heures à l'usage de Rome (Heures Tabourot) conservées à l'Université McGill, Livres rares et collections spécialisées, MS 154 dans son article « De quelques livres d'Heures remarquables... », en particulier pages 257-262. De plus signalons que dans le cadre du Catalogue des livres d'heures conservés au Québec, B. Dunn-Lardeau, en collaboration avec G. Samson, prépare la notice codicologique de ce livre d'heures. B. Dunn-Lardeau distingue deux mains à l'œuvre dans ce manuscrit : celle du Maître des Prélats Bourguignons et celle d'un assistant.

8. Voir le livre d'heures dit « Berbisey Hours » qui s'est vendu à Londres, Christie’s, 11 juillet 2002, lot 38, peint par le Maître des Prélats bourguignons et acquis en 2003 par la Bibliothèque municipale de Dijon (Ms. 3765). Le commanditaire est peut-être Étienne II Berbisey, vicomte-mayeur de Dijon ou Thomas, secrétaire des Finances de Bourgogne (mort en 1533). Citons les travaux de M.-F. Damongeot-Bourdat, «Un livre d'heures inédit de la famille Berbisey », Art de l'enluminure 13 (2005) : 8-39.

9. Parmi les saints rajoutés, on signalera, en latin, en janvier : Emerentianae ; Convers[io] S. Pauli ; Valerii ; Potentianae ; en février : Alexii ; Gabrielis ; en mars : Mariae Aegipt. ; en avril : Ioachimi ; Petri Martiris ; en mai : Helenae ; Desiderii ; en juillet : Theobaldi ; Benedicti ; Henrici ; Bonventurae ; Alexii ; Apollinaris ; en août, Dominici ; Rochi (d'une autre main) ; Ludovici ; en novembre : Caroli Borromei (d'une autre main ; la même que Roch, en août ; notons que Charles Borromée, archevêque et cardinal, fut canonisé en 1610); en décembre : Barbarae virg. ; Nicolai ; Conceptio B. Mariae. On notera l'insertion de saint Apollinaire en juillet au calendrier : Guillaume Tabourot était seigneur de la Tour Saint-Apollinaire. 
peu plus le livre de piété où sont consignés les dates importantes d'une famille déjà illustre.

\section{Un livre de raison autographe : Guillaume Tabourot, seigneur de la Tour Saint-Apollinaire (1573-1644)}

L'essentiel de ce livre de raison est copié par une même main, bien assurée, celle de Guillaume Tabourot ${ }^{10}$. Nous conservons, grâce aux travaux et sondages de Marie-Françoise Damongeot-Bourdat, un document certainement autographe de la main de Guillaume Tabourot. Il s’agit d'un reçu, signé « Tabourot », daté du 6 décembre $1634^{11}$. Un dessin (collection particulière) reproduit un portrait de profil de Jean-Antoine de Baif (1532-1589) dans un médaillon : il est signé et daté « Pingebat Guill. Taborotius. 1594 » et on y reconnaît clairement la main de notre livre de raison, avec le tracé des lettres « $T$ » ou « $G$ » majuscules très caractéristiques (Voir fig. 2) ${ }^{12}$.

10. On signalera que Guillaume Tabourot rajoute également au feuillet $126 \mathrm{r}^{\circ}$ une oraison à la Vierge en latin, copiée dans une écriture un peu plus italique, trahissant sans doute une admiration ou un goût pour les calligraphies italiennes qu'il aurait admiré lors de son voyage en Italie cité au feuillet $126 \mathrm{v}^{\circ}$. Son écriture elle-même dans le livre de raison est imprégnée d'un certain italianisme.

11. Ce reçu est reproduit dans Henri-Stéphane Gulczynski, «Les Tabourot et l’architecture. Architecture, amateurisme et société à Dijon du début du XVIe siècle au milieu du XVIIe siècle», in Tabourot, seigneur des Accords. Un Bourguignon poète à la fin de la Renaissance, dir. François Moureau et Michel Simonin (Paris : Klincksieck, 1990), 41 planche 5. Le document fut signalé à H.-S. Gulczynski par Mme MarieFrançoise Damongeot, alors conservateur à la Bibliothèque municipale de Dijon.

12. Daté 1594, Guillaume Tabourot est alors jeune (21 ans) : ce dessin révèle un véritable talent de dessinateur. Sur le pourtour du médaillon figure le nom en capitales " FRANCOIS + IEAN + ANTHOINE + DE + BAIF + POETE ». Jean-Antoine de Baïf était ami de Ronsard et membre de la Pléiade. Ce dessin prend pour modèle la gravure (portrait en médaille à l'antique) placée en tête de l'édition de 1572 des œuvres de J.-A. de Baif : [CEuvres en rime] Les Amours, 4 vols. (Paris : Lucas Breyer, 1572) [première édition collective des œuvres de De Baif]. Le médaillon est monté face au titre. On citera également la gravure signée G. C. F. qui paraît pour la première fois figure sur la page de titre des Mimes de 1576, puis de nouveau dans les Mimes, enseignemens et proverbes, A Tolose, pour Jean Jagourt [sic], 1608 [1605] (Jean-Anthoine de Baif, Mimes, enseignemens et proverbes, éd. Jean Vignes [Genève : Droz, 1992], 44). Une illustration de la version gravée de ce dessin est reproduite dans le catalogue de vente Paris, Sotheby's, 31 mai 2016, lot 6, p. 11. 

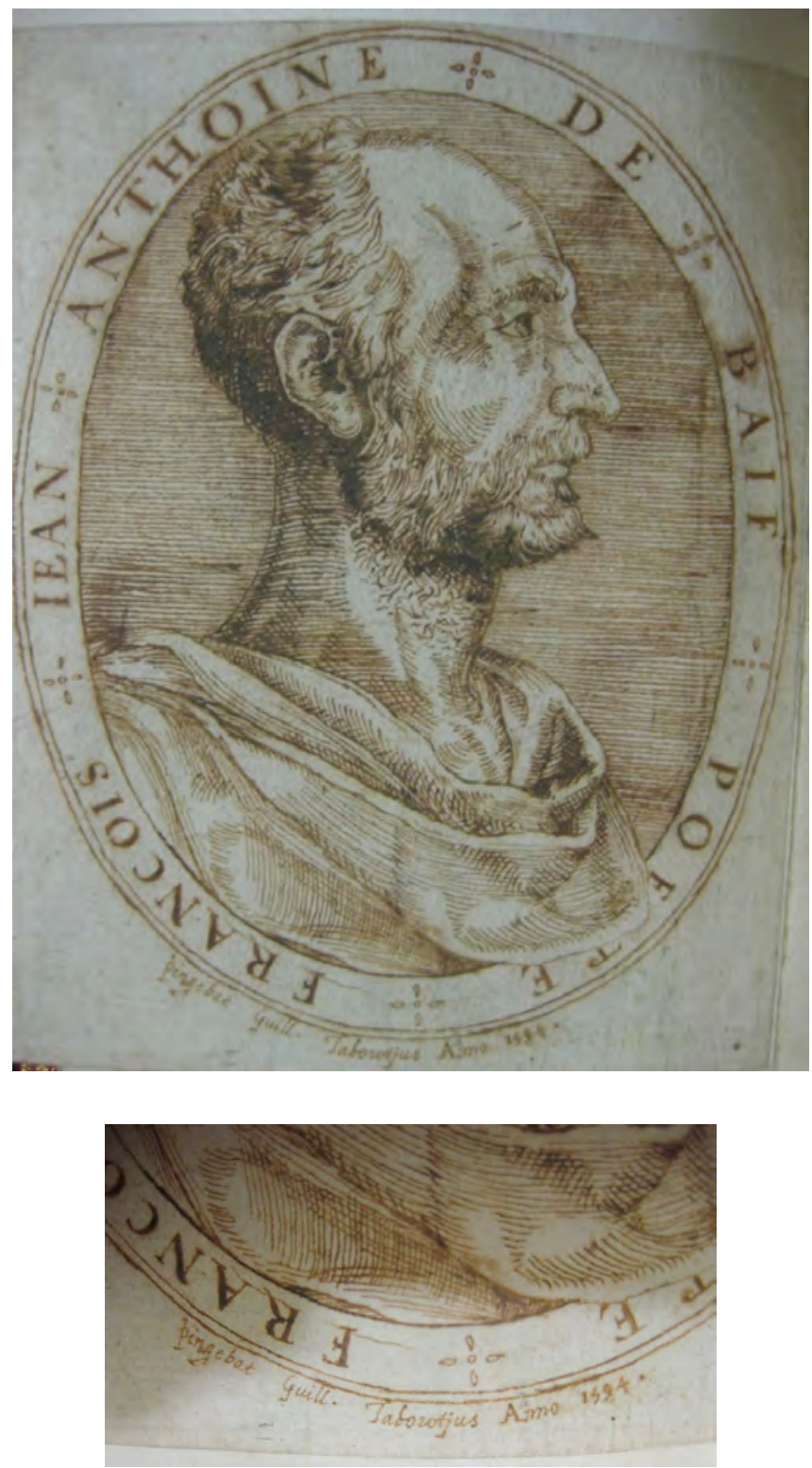

Fig. 2. Dessin à l'encre figurant Jean-Antoine de Baï, signature autographe de Guillaume Tabourot, 1594. Copie d'un portrait gravé et détail de la signature. France, collection particulière. 
La copie du livre de raison contenu dans les Heures McGill, MS 154 trahit une écriture des plus régulières, manifestement sans interruption dans la copie (avec un texte qui ne semble pas couché sur papier au fur et à mesure des évènements). Ceci suggère la mise au propre du livre de raison, sans doute un temps noté et tenu à part, et ici reporté dans le livre d'Heures de famille. Les naissances, les trépas et les évènements notables se sont déroulés entre 1606 et 1631, et la copie ou mise au propre a dû être faite peu de temps après 1631. Guillaume Tabourot meurt en 1644 : pendant les quinze dernières années de leur mariage, le couple n’a pas enregistré ou rajouté d'entrées. On relève à la fin des feuillets - après la mort de Guillaume Tabourot - quelques entrées copiées par sa femme Jeanne Bernard, d'une écriture et orthographe nettement moins assurée (fol. 127 $\mathrm{v}^{\circ}$ ), inscrivant la mort du pater familias Guillaume Tabourot en 1644, suivie de la naissance d'une petite-fille Jeanne Tabourot en 1645. Puis, ce manuscrit dût passer peut-être chez l'un des enfants de Guillaume et Jeanne : un document d'archives nous renseigne sur les modalités de partage de la bibliothèque de Guillaume Tabourot entre sa veuve Jeanne Bernard et Théodecte Tabourot, leur fils né en 1609 et chanoine de la cathédrale de Langres ${ }^{13}$.

Ce livre de raison donne à voir les liens étroits qu'entretenaient les «gens de basoche » avec le milieu parlementaire en ce XVII siècle à Dijon, tous issus de la haute caste robine humaniste bien dotée en bibliothèques et sensible aux travaux d'érudition et d'architecture ${ }^{14}$. Le père de Guillaume, Étienne Tabourot dit seigneur des Accords - plus célèbre comme homme de lettres — était déjà

13. Voir le document suivant : arrangement successoral daté du 19 octobre 1644. Dijon, Archives départementales de la Côte-d'Or, notaire P. Camus, $n^{\circ} 120$. Notons que Guillaume Tabourot y est qualifié de "maître des requêtes de feu la reine mère ». Ce document est cité par Henri-Stéphane Gulczynski, «Les Tabourot et l'architecture... », 63 et Henri-Stéphane Gulczynski, « La construction du château de Cormatin. Nouvelles approches sur l'historique des travaux, à propos de documents inédits relatifs à Guillaume Tabourot », in Bulletin monumental 154.1 (1996), note 29.

14. D’autres livres de raison issus de ce même milieu pourront être consultés et comparés, tel celui non localisé, mais connu, d’Étienne Bernard (voir note n 25) ou encore celui de Pierre Mareschal (né en 1544), conseiller du roi et président de la Chambre des comptes de Dijon. Pierre Mareschal était le neveu de François Mareschal, un contemporain connu d'Étienne Tabourot, à qui le littérateur dédia le second chapitre du quatrième livre des Bigarrures. Ce livre de raison a été édité par Noël Garnier dans le Bulletin d'histoire et d'archéologie religieuses du diocèse de Dijon XII (1894) : 153-159. Le livre de raison est inséré dans les Heures de Pierre Mareschal, conservées à Melbourne, State Library of Victoria, 96 / R66 Hm. On consultera l'étude de Keith Val Sinclair, « Un manuscrit enluminé ayant 
bien établi à Dijon où il exerça sa charge d'officier du roi en tant que procureur du roi au baillage de Dijon depuis 1582 et capitaine de la paroisse de Saint-Jean depuis 1574. Son fils aîné Guillaume II Tabourot briguera à Dijon également des charges administratives. Il sera qualifié très jeune d'avocat en 1591. S'il dut renoncer à l'office de procureur du roi au bailliage et chancellerie de Bourgogne en 1595, il deviendra "conseiller maître ordinaire des requêtes de l'hôtel de la reine-mère et du duc d'Orléans » vers 1611, puis bailli de Bellegarde (aujourd'hui la ville de Seurre) en 1619. En 1611, la reine-mère était Marie de Médicis, veuve d'Henri IV assassiné en 1610 et donc mère de Louis XIII mineur. Le duc d'Orléans était alors le troisième fils d'Henri IV, à savoir Gaston d'Orléans (1608-1660), fait duc au moment de la mort de Nicolas en 1611, second fils du roi. Guillaume Tabourot est donc proche de cette cour royale où il côtoiera architectes, poètes et haute société.

Un certain nombre d'historiographes ont voulu voir en Guillaume II Tabourot un architecte ${ }^{15}$. Si les Tabourot, père et fils, ont tous deux certainement présenté un intérêt pour l'architecture, il semble que l'on ne conserve aucun document dans lequel Guillaume II Tabourot soit désigné explicitement comme " architecte ». L'article de H.-S. Gulczynski (1990) est éclairant à cet égard ${ }^{16}$. Ses sondages dans les archives des notaires font apparaitre que des personnages publics comme Guillaume Tabourot, Étienne Bouhier ou Pierre Desbarres donnent un avis dans le cadre de reconstructions ou de modifications architecturales, mais ne sont en aucun cas les architectes ou maitres d'œuvre

appartenu à deux bourguignons, Nicole Fonssard et Pierre Maréchal », Annales de Bourgogne, 34.135 (1962) : 170-178.

15. Voir l'ouvrage de Marcel Mayer, Une famille d'artistes bourguignons. Les Tabourot architectes (Dijon : Imp. Régionale, 1907) ; cette affirmation fut reprise dans plusieurs articles et notices bibliographiques. Un recueil de plans et de dessins d'architecture acquis par la Bibliothèque municipale de Dijon (ms. 1654) attribuait à Guillaume Tabourot un certain nombre de bâtiments et constructions à Dijon, dont les plans de certains hôtels particuliers (Hôtel Desbarres, Hôtel Baillet de Vaugrenant), plusieurs couvents de Dijon (ceux des Carmes, des Carmélites et des Ursulines) et certaines parties de châteaux en Bourgogne ou en Franche-Comté (par exemple le château de Cormatin, en Saône-et-Loire. Sur ce dernier, voir Gulczynski, « La construction du château de Cormatin... », 25-38).

16. « Une tradition familiale d'intérêt pour l'architecture est bien à mettre à l'actif des Tabourot. Nous relèverons au passage un témoignage assez éloquent de cet intérêt de la part d'Étienne - pourtant peu souligné en général. Mais cette tradition n’a peut-être pas correspondu tout à fait avec ce que l'on aurait désiré [...] Ni Pierre, ni Guillaume ne sont jamais désignés comme architectes dans les textes d'époque connus à ce jour », Gulczynski, « Les Tabourot et l'architecture... », 35. 
des chantiers en question (il donne l'exemple des Ursulines de Dijon) ${ }^{17}$. Ces notables, investis dans les constructions locales, seraient plutôt des sortes de conseillers et bienfaiteurs d'un couvent, d'un hôtel ou autre bâtiment, apportant en quelque sorte leur caution publique aux nouveaux bâtiments. Toutefois, les travaux plus récents de H.-S. Gulczynski viennent tempérer cette remise en question et font de Guillaume II Tabourot un « architecte amateur » qui joua un rôle essentiel dans l'élaboration du programme architectural du château de Cormatin (Saône-et-Loire), mais aussi de bâtiments tels les Ursulines (Dijon). Tabourot fut proche de l'architecte jésuite Étienne Martellange ${ }^{18}$. Il semble, en fait, pour reprendre une expression de H.-S. Gulczynski, que comme Étienne Bouhier son ami, Guillaume Tabourot ait été plutôt un « responsable de projet », définissant une partie d'un programme architectural, mais en aucun cas maître d'œuvre ou exécutant ${ }^{19}$.

\section{Vers une reconstitution des bibliothèques Tabourot : père, oncle, frères et fils}

La présence d'un livre de raison dans un manuscrit de la qualité des Heures McGill, MS 154 pose le problème de la (ou plutôt des) bibliothèque(s) d'une famille comme celle des Tabourot. Comme cela a été déjà évoqué, nous savons qu'Étienne Tabourot possédait une bibliothèque, dont son fils aîné Guillaume a dû hériter en grande partie ${ }^{20}$. À sa mort, ce dernier avait toujours

17. Gulczynski, «Les Tabourot et l'architecture... », 49 et 53. Les documents stipulant que Guillaume Tabourot et les autres notables apparaissent comme « ayant donné un avis » dans la construction de bâtiments pour les Ursulines de Dijon sont conservés dans la liasse AD de la Côte-d'Or : notaire Philippe Camus, $\mathrm{n}^{\circ}$ 131. Le couvent des Ursulines reçoit les filles de Guillaume Tabourot, Pierre Desbarres et Charles Fevret, comme le stipulent les actes passés devant le notaire Camus et contenus dans la même liasse.

18. Gulczynski a étudié en 1996 le château de Cormatin (Saône-et-Loire) construit pour Antoine du Blé, baron d'Uxelles et son fils Jacques. Voir Gulczynski, « La construction du château de Cormatin... », et tout particulièrement sur Guillaume Tabourot, page 32 : «Cependant, ce dernier [Guillaume Tabourot] semble avoir été débordé par toutes ses fonctions ». Voir note $n^{\circ} 77$ ci-dessous pour un dessin du château de Saint-Applume (propriété de Guillaume Tabourot) signé Étienne Martellange.

19. Gulczynski, « La construction du château de Cormatin... », 32.

20. Gilles Banderier signale deux livres ayant appartenu au littérateur Étienne Tabourot. Voir Gilles Banderier, Bibliothèque d'humanisme et Renaissance 59 (1997) : 335 ; Gilles Banderier, Nouvelles du livre ancien, 101 (2000) : 4 . 
une bibliothèque conséquente ${ }^{21}$ : un document d'archives fait état d'un arrangement successoral en 1644 entre Jeanne Bernard, sa veuve et Théodecte Tabourot, son fils, chanoine de la cathédrale de Langres ${ }^{22}$. Un livre conservé à Yale (Beinecke Library) semble suggérer que les enfants d'Étienne avaient hérité de la bibliothèque de leur père, ou du moins possédaient en commun un livre, avec un ex-libris au titre qui indique : "Gulielmi et Petri filiorum Steph[ani] Taborotii » (voir fig. 3) ${ }^{23}$.

Le présent livre d'Heures pouvait lui venir soit de la famille Tabourot ou de celle de sa femme au moment du second mariage (le livre de raison commence précisément l'année de leur mariage, ce qui ne doit pas être un hasard), soit comme acquisition ou cadeau de noces. On ne peut pas non plus exclure que le livre d'Heures faisait peut-être partie de la bibliothèque d'Étienne Tabourot, mais si cela avait été le cas, sans doute le seigneur des Accords aurait fait apposer son ex-libris, comme dans un certain nombre de livres qui le contiennent ${ }^{24}$. De plus, si le livre d'Heures avait déjà été conservé au sein de la famille Tabourot, et qu'il était passé par descendance à Guillaume Tabourot, il est raisonnable

21. À l'instar de celle de son grand ami Étienne Bouhier, voir l'étude d'Albert Ronsin, La Bibliothèque Bouhier: Histoire d'une collection formée du XVI e au XVII e siècle par une famille de magistrats bourguignons, Mémoires de l'Académie des Sciences, Arts et Belles-Lettres de Dijon, tome CXVIII (Dijon : Imprimerie Bernigaud et Privat, 1971). Voir également la note $n^{\circ} 64$.

22. Voir note $\mathrm{n}^{\circ} 13$ ci-dessus.

23. Ex-libris : « Guillaume et Pierre, fils d'Étienne Tabourot ». Yale, Beinecke Library, Eq21 564A : Apian, Cosmographia Petri Apiani, / per Gemmam Frisium apud Louanienses medicum \& mathematicu[m] insignem... (Anvers : héritiers d'Arnold Birckmann, 1564).

24. Dans l'attente d'une étude ou du moins d'un recensement des livres ayant un temps appartenu à Étienne Tabourot, seigneur des Accords et auteur des Bigarrures, citons par exemple Antoine de la Sale, Paradis de la reine Sibylle, manuscrit vers 1440, exemplaire dédié à Agnès de Bourgogne, puis doté de l'ex-libris (daté 1576) et de la devise d'Étienne Tabourot au fol. 1 (Chantilly, Bibliothèque Condé, 653); Jacques Milet, Histoire de la destruction de Troie, qui porte la mention suivante : «A Tabourot, du don de Monsieur le baron de Lux. 1590 » (Genève, Bibliothèque de Genève, MS. fr. 177, voir Bibliothèque de Genève, Catalogue des manuscrits français [1-198] [Genève, 2011], 317) ; ou encore dans l'ouvrage Spectaculorum in susceptione Philippi Hisp. Prin. divi. Caroli V... (Anvers, 1550) : "Stephani Taborotii sum. A tous accords » (Vente, Paris, Hôtel Drouot, 12-17 juin 1882, Catalogue illustré des livres précieux manuscrits et imprimés faisant partie de la bibliothèque de M. Ambroise Firmin-Didot, 222-223, no 485). Un embryon de recensement a été entrepris par Banderier, "Ex bibliotheca Taborotiana ", 437-440, citant deux imprimés - Du Bellay (Paris, 1558) et Aulu-Gelle (Lyon, 1565) - comportant l'ex-libris d'Étienne Tabourot. 


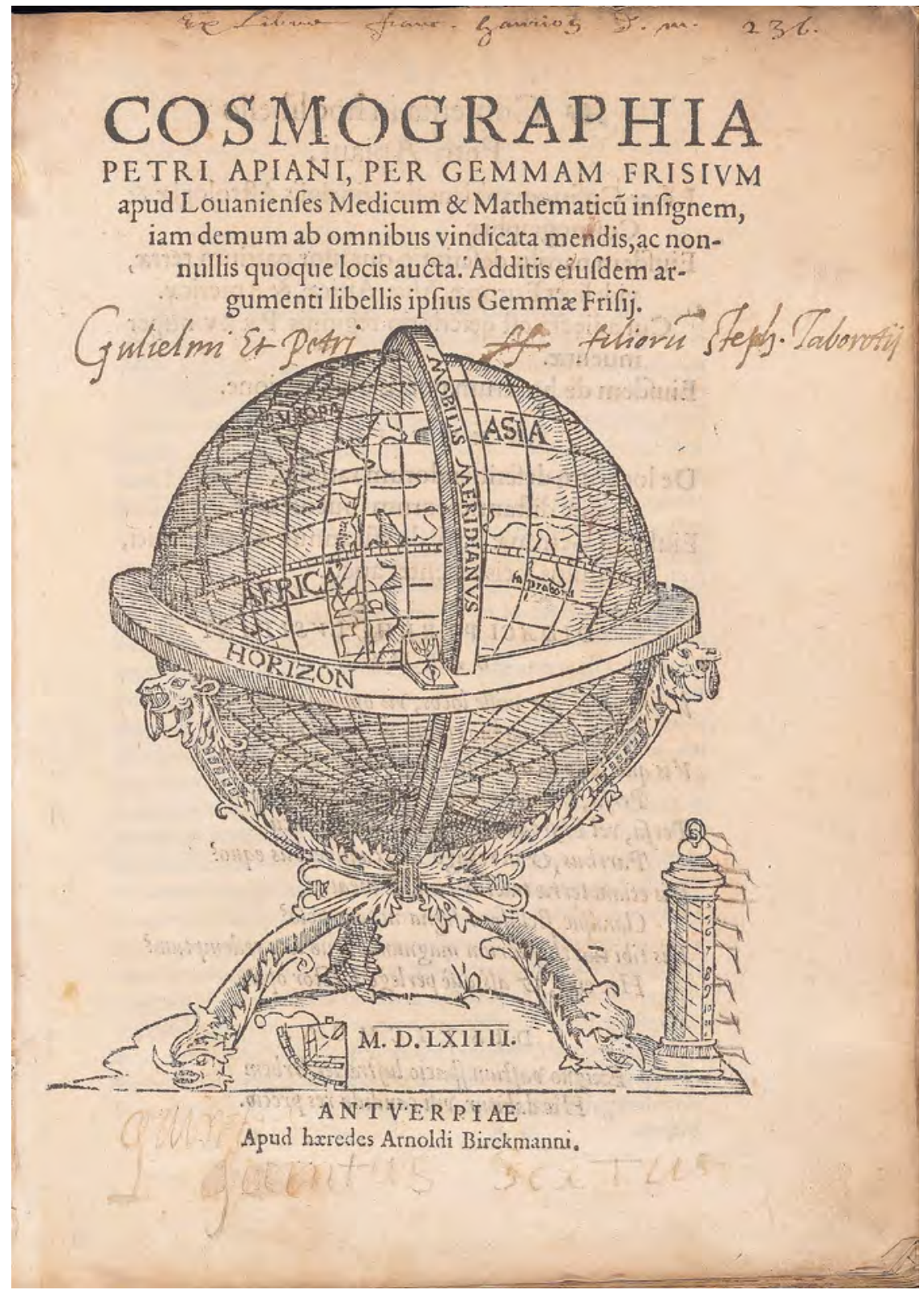

Fig. 3. Ex-libris des frères Guillaume et Pierre Tabourot, fils d'Étienne Tabourot. Yale, Beinecke Library, cote Eq21 564A. 
de penser que Guillaume Tabourot y aurait consigné les temps forts de son existence avant 1606 et notamment son premier mariage avec Louise Rougette.

Par ailleurs, nous savons qu'Étienne Bernard, beau-père de Guillaume Tabourot, tenait un livre de raison. Ce livre a depuis disparu ou du moins n’est pas localisé. Le livre de raison d'Étienne Bernard est néanmoins cité par le Commandeur de Broqua dans son ouvrage consacré à Claude Bernard, le "Pauvre prêtre ": "Il ne peut y avoir aucun doute sur la date et le lieu de sa naissance, car Étienne Bernard a rédigé de sa main le livre de raison de sa famille, sur les premiers feuillets du splendide missel dont il faisait usage, et que la famille de Sassenay conserve aujourd'hui avec un filial respect $»^{25}$. Un autre livre d'Heures a appartenu aux Tabourot : citons un livre d'Heures copié et peint à Bruges, avec curieusement les armoiries des Visconti (les premiers commanditaires), auquel on a rajouté un ex-libris, "Theodectis Taboroti sum». Meurgey, dans son étude consacrée à ce manuscrit, indique qu'il doit s'agir de Théodecte Tabourot, chanoine de Langres et frère d'Étienne Tabourot des Accords, littérateur ${ }^{26}$.

Une recherche plus développée sur les bibliothèques des différents membres de la famille Tabourot s'impose et sera des plus instructives, ne serait-ce pour distinguer les différents membres de la famille, dont les multiples «Théodecte $»^{27}$.

25. Le Commandeur de Broqua écrit en 1914 : Joseph Jean de Broqua, Claude Bernard dit le Pauvre Prêtre (1588-1641) (Paris : Lethielleux, 1914). Depuis, les archives de la famille Bernard de Sassenay ont été déposées en 1974 à la section des Archives privées aux Archives de France sous les cotes 337 AP/1 à $337 \mathrm{AP} / 38$. La famille Bernard de Sassenay est originaire de Bourgogne et ce sont les descendants d'Étienne Bernard, député du Tiers État de Bourgogne. Pour l'heure, un premier examen de ces archives n'a pas permis de retrouver le livre d'Heures de la famille Bernard ni son livre de raison.

26. Ce manuscrit fut légué à l'Hôpital de Tournus (Saône-et-Loire) en 1929 par la baronne de Laduye. Il est dit dans l'étude que le manuscrit a appartenu à Théodecte Tabourot puis passa dans la branche Tabourot de Véronnes: Jacques Meurgey, Les Heures de Tournus. Étude sur un manuscrit du XV siècle aux armes des Visconti conservé à l'Hôpital de Tournus (Mâcon : Protat frères, 1930), 14-15).

27. Nous savons qu'un certain nombre de livres ont aussi appartenu à un dénommé Théodecte Tabourot, qui peut être un frère d'Étienne Tabourot, ou bien un fils d'Étienne Tabourot, ou encore un fils de Guillaume II Tabourot. Citons, par exemple, ce recueil de dessins copiés sur des estampes du graveur Énea Vico (Paris, BnF, Estampes, Te-21-4) : on lit à la dernière page, écrit au crayon, "C'est A Moy Theodecte Tabourot », sans pouvoir identifier de quel « Théodecte » il s'agit. 


\section{Édition du livre de raison de Guillaume Tabourot et Jeanne Bernard, années 1606-1645}

Outre l'état civil et des archives souvent disparates, les livres de raison constituent un document fiable pour établir les naissances, les décès et les relations sociales tissées au sein d'une communauté, telle celle des magistrats et hommes publics de la Bourgogne des XVI et XVII ${ }^{e}$ siècles. Au-delà de la fiabilité des informations contenues, de son importance historique et généalogique, un livre de raison est un document émouvant, conservé dans un ouvrage de prix, parfois hérité de père en fils, de mère en fille. Sa valeur artistique est doublée pour la famille concernée - ici celle des Tabourot - d'une valeur sentimentale, témoignant aussi de la fierté de son lignage, des relations établies avec les autres familles dijonnaises, chalonnaises et langonaises. À travers un document comme celui-ci, il se confirme qu'à Dijon, à Chalon-sur-Saône, à Langres, les mêmes offices étaient entre les mains des mêmes familles et que ce réseau passait souvent par des mariages, mais aussi par l'honneur de tenir un enfant sur les fonts baptismaux. Les Tabourot, comme toutes les familles égrenées le long des feuillets du livre de raison, jouèrent un rôle considérable dans les manœuvres politiques et religieuses de Dijon, capitale bourguignonne, et des villes avoisinantes. Ces générations de robins étaient souvent des docteurs en droit et fondateurs de lignées qui allaient, socialement et intellectuellement, primer et diriger la société bourguignonne durant plus de deux siècles. Par-delà leurs liens sociaux, ils partageaient des liens culturels, effectuant à plusieurs, par exemple, le voyage incontournable et formateur en Italie ${ }^{28}$ : « Le 6 septembre suivant, je sortis de cette ville pour mon voyage d'Italie avec Monsieur Bernard mon beau frere et en retournames le 6 d'apvril suivant ». Notre livre de raison révèle que ce voyage fut relativement long (sept mois). Il ne confirme pas la

28. Sur le voyage en Italie au XVII ${ }^{\mathrm{e}}$ siècle, on consultera François Brizay, Touristes du grand siècle. Le voyage d'Italie au XVII siècle (Paris : Belin, 2007). Brizay cite le géographe et voyageur Pierre Duval : "Le Voyage d'Italie a tant de charmes, qu'il est difficile de s'en dispenser : il est ordinairement préféré à tous les autres et toutes les Nations de l'Europe tombent d'accord que l'on n'a pas veu de beau pays si l'on n'a pas veu l'Italie » (page 5). Il est intéressant de noter que le voyage en Italie constitue un des seuls évènements hors naissances, baptêmes et décès que Guillaume Tabourot choisit d'inclure dans son livre de raison. Ce voyage devait — pour un homme de son érudition et de sa condition sociale — représenter un des temps forts de son existence, suffisamment pour figurer dans son livre familial. Le seul autre évènement non familial retenu par Guillaume Tabourot sera l'entrée du roi Louis XIII à Dijon en 1629 (voir ci-dessous, note $\mathrm{n}^{\circ} 74$ ). 
présence d'autres co-voyageurs, mais la tradition veut que Guillaume Tabourot entreprît ce voyage avec Étienne Bouhier ${ }^{29}$.

Le rôle culturel de la famille Tabourot était déjà bien confirmé à travers les personnalités d'Étienne, de Guillaume et d'autres membres de la dynastie, en amont et en aval. Mais leur rôle et prestige social, les relations avec le milieu dijonnais et bourguignon plus étendu est - grâce à ce livre de raison - désormais un peu mieux précisé.

29. Voir Gulczynski, « La construction du château de Cormatin... », 38, note 57 : « Selon une tradition familiale, Étienne Bouhier, qui fit construire l'hôtel de Vogüé et fonda l'Hôpital général avec G. Tabourot, aurait fini ses études à Padoue et aurait fait le tour d'Italie avant d'entrer au Parlement de Dijon en 1607 ». Voir aussi Ronsin, 22-23. 


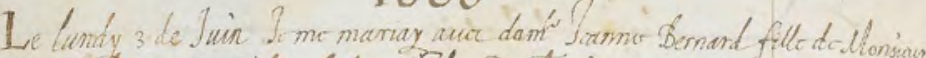

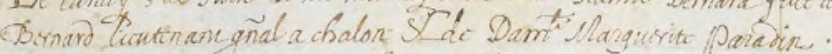

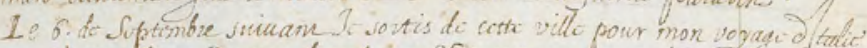

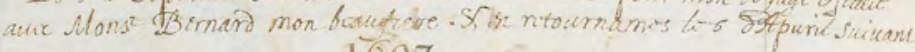

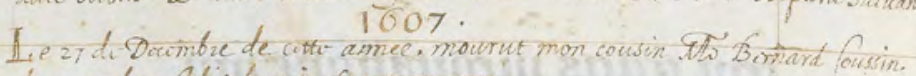

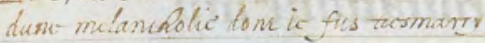

Le 24 de Sanvia bos naquir Gabridle a 4 how. vn quan apro midy \& 1605

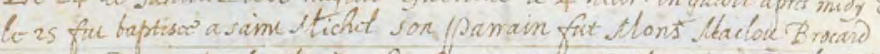

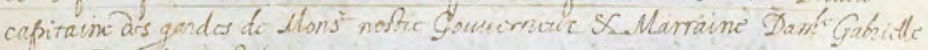
Chiquoi d Monpaste ma mire.

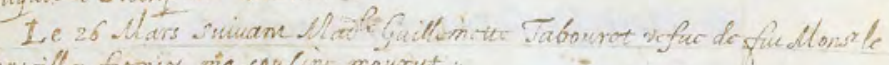
Conscille formio ma con ine mourut

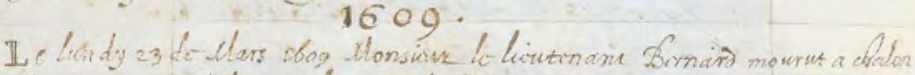
plaine or raprotte do tous les gins de bien.

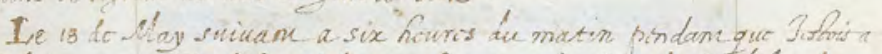
Chalon naquic Thodich Taborwot. fon parrein fut llonst toficial mon

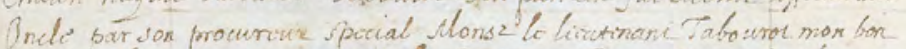
Cousin Lux a Marrine that Catharine Jurt fommo de Mlonsese

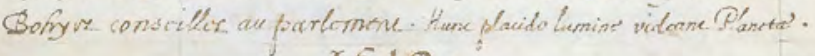

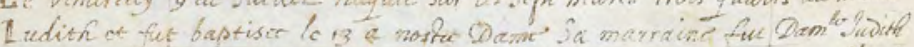

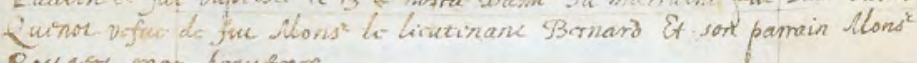
Rougcal mon bcaufrere.

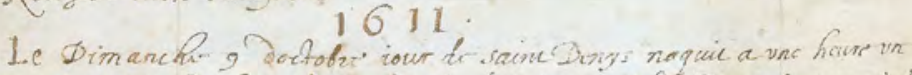
quart asres midy Iequeline \&t fut baptisce a Yosfor Dame Sa marnine'

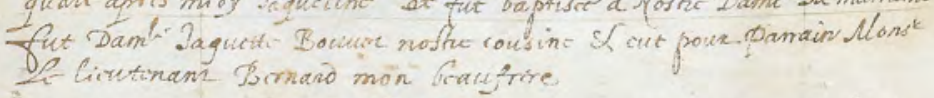

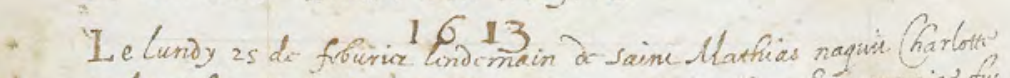
a dux ficun's inuiron quin? minuts afres midy. Sa marraine fue

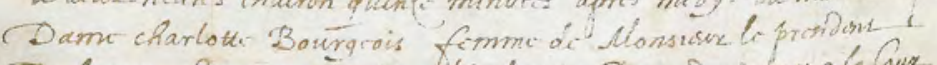
Desbarres. Et son parrain tot. elaude Birnaze adubcac a la fur son onde matonild.

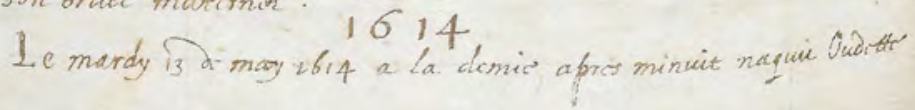

Fig. 4. Livre de raison de Guillaume Tabourot et Jeanne Bernard, années 1606-1614. Autographe de Guillaume Tabourot. Montréal, McGill, LRCS, MS 154, fol. 126vº. 


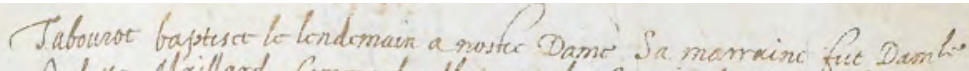

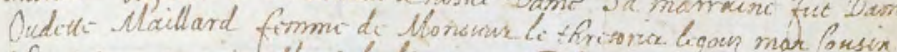

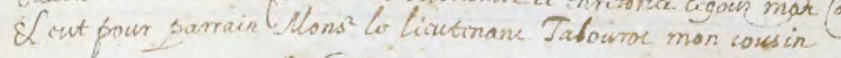
1615

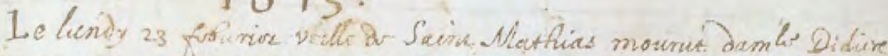

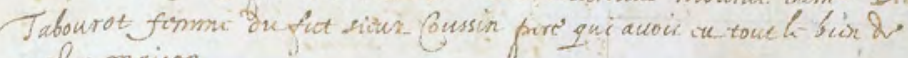
nostu maison

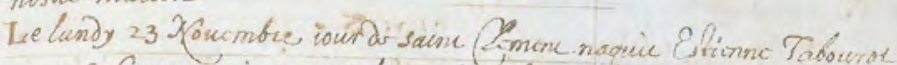

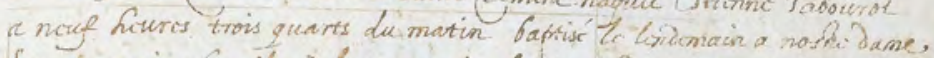
Son farrain fue thons le serrtain Arriset of sa marrainc Dambo

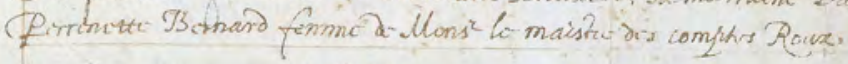

I. mardy Jour de Joustit.

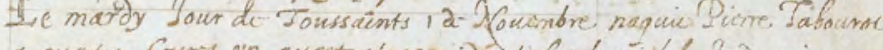

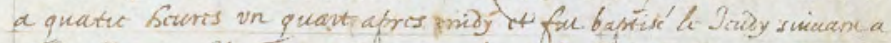

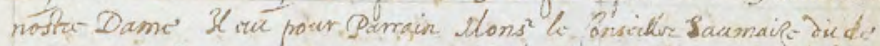

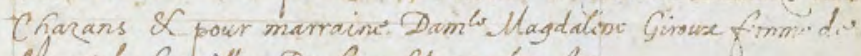
Nons: le fonsither Bouchyor fils mon bon Atmy.

\section{161919.}

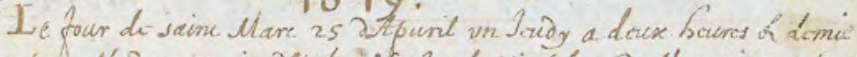

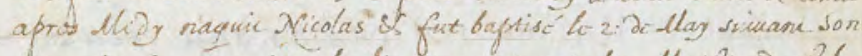
pamain fut monsisiz le livitinane pariculir llathica a colalon pour liguel moni poux prista la maix Et sx mamaine fut

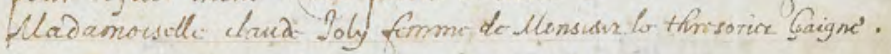

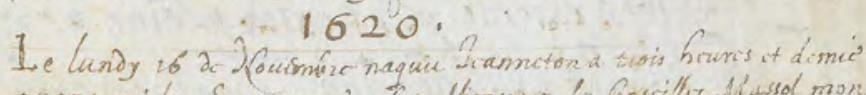

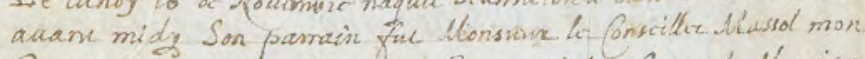
Cusin R sa Mamaine Dame Zurme Vadot funme de thorriner. ta prisian Girouxe of fur Gaptise? te maroy a most. Dam?.

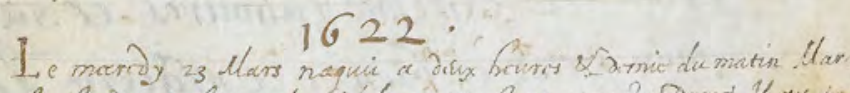

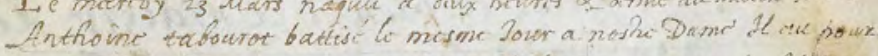

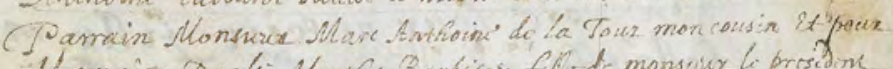

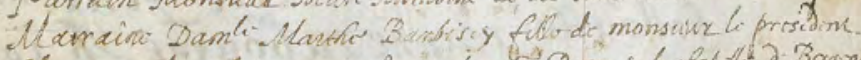

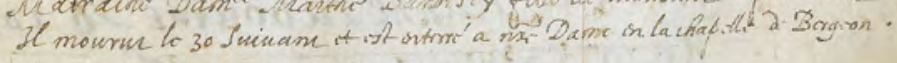

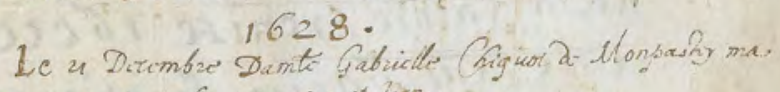

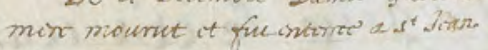

Fig. 5. Livre de raison de Guillaume Tabourot et Jeanne Bernard, années 1614-1628. Autographe de Guillaume Tabourot. Montréal, Université McGill, LRCS, MS 154, fol. $127 \mathrm{r}^{\circ}$. 


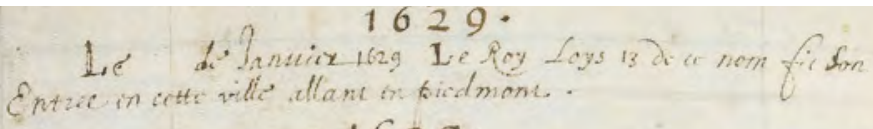

1. edmanin 232 ? 1630 .

to wirte Clicure it thand

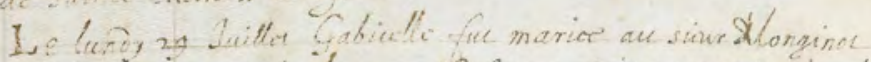

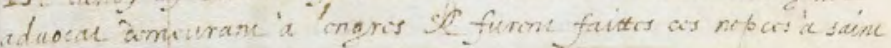
Aflomet

\section{1}

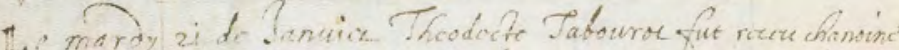

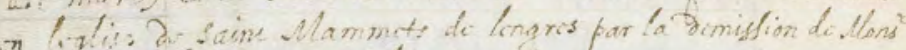

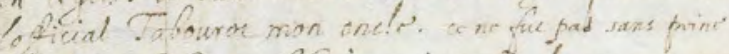

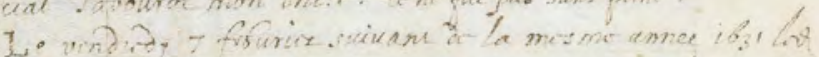

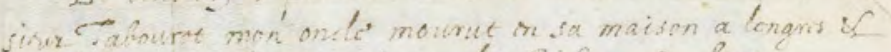

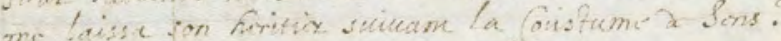

\section{Gceciini}

-c sammedin dixzeme descrfombre mon cher mavip moureu cheres enfe frewte bs a - a menuis soudcmane dieu narsa bonee luy

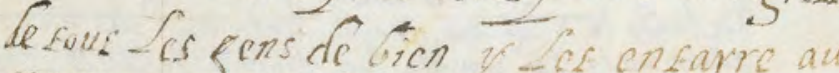
EQUerant nere iacounin thu vsent Aemande solon fil cricione sabouros cF safamme mon faice ce bicn er ce raisir de man moic Lerer nctife fille ma filloule icantie fabouros le iour Ca fa sainfe Ercnife 1 bccctu che frue bakise le ctili icun

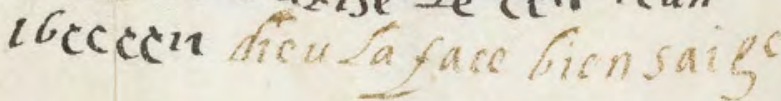

Fig. 6. Livre de raison de Guillaume Tabourot et Jeanne Bernard, années 1629-1645. Deux mains : autographe de Guillaume Tabourot et ajouts de Jeanne Bernard. Montréal, McGill, LRCS, MS 154, fol. 127vº. 


\section{Édition $^{30}$ du livre de raison (années 1606-1645) de Guillaume II Tabourot (1573-1644), fils d'Étienne Bernard, dit Tabourot des Accords auteur des Bigarrures (1572) et Jeanne Bernard, fille d'Étienne Bernard, ligueur puis lieutenant général du baillage de Chalon-sur-Saône}

[fol. 126 $\mathbf{v}^{\circ}$ ]

\section{6}

Le lundy 3 de juin je $\mathrm{e}^{31}$ me mariay avec damoiselle Jeanne Bernard ${ }^{32}$ fille de monsieur Bernard $^{33}$, lieutenant general à Chalon et de damoiselle Marguerite Paradin ${ }^{34}$. Le 6 de septembre suivant je sortis de cette ville pour mon voyage d'Italie avec monsieur Bernard mon beau frere ${ }^{35}$ et en retournames le 6 d'apvril suivant ${ }^{36}$.

30. Les principes d'édition ont été les suivants : quelques mots ont fait l'objet d'une note soit pour leur sens, soit pour leur forme. Nous avons accentué les finales en -ée (née, année) et restitué l’accent grave sur les lettres $a$, e et $u$ dans les prépositions et les adverbes monosyllabiques pour les distinguer des mots homographes ( $\grave{a}$, là, dès, lès, ò̀). En revanche, nous n’avons pas accentué la lettre e à l'intérieur d'un mot (pere, frere). Les abréviations ont été développées, par exemple monsr (monsieur), me (maître).

31. Guillaume Tabourot (1573-1644), fils d'Étienne Tabourot (1549-1590). Guillaume Tabourot épousa Jeanne Bernard en 1606. Gulczynski cite une archive conservée aux AD de la Côte-d'Or : notaire Philippe Camus, no 97 (Gulczynski, « Les Tabourot et l'architecture... », 49). Ce sont, pour Guillaume Tabourot, des secondes noces : celui-ci avait épousé en premières noces Louise Rougette, dont il aurait eu un fils Jean, baptisé à Saint-Michel le 20 avril 1596 et qui mourut en bas âge (voir Perrenet, 23).

32. Jeanne Bernard, fille d'Étienne Bernard et de Marguerite Paradin. Henri Drouot la dit née en 1582 (il cite un acte de baptême daté 7 juillet 1582, Dijon, Archives départementales de la Côte-d'Or, B 494, fol. 121 ; voir Henri Drouot, « Un père de famille sous Henri IV. Lettres domestiques d'Étienne Bernard (1598-1609) ", Annales de Bourgogne 24 [1952] : 160-175). Jeanne Bernard était donc âgée de 15 ans et demi lors du premier veuvage de son père Étienne Bernard (Marguerite Paradin, sa mère meurt en 1597 ; voir Drouot, 163). Le contrat de mariage est conservé à Dijon, AD de la Côte-d'Or, notaire Philippe Camus, $\mathrm{n}^{\circ}$ 97, $1^{\text {er }}$ juin 1606, avec liste des témoins. Si l'on en croit l'article d'Henri Drouot, Étienne Bernard fut, une fois ce mariage conclu, beaucoup moins tourmenté par l'isolement de ses filles (Jeanne, Élisabeth, Pernette) et de son fils Claude dit « le Pauvre Prêtre » : «À partir de ce moment-là, la présence de son gendre, Guillaume Tabourot, homme d'expérience, auprès de ses filles et de son deuxième fils, atténuera beaucoup ses préoccupations » (Drouot, 164). Les lettres d'Étienne Bernard à sa fille avaient été rassemblées par Théodecte Tabourot, chanoine de Langres (né en 1609, cité dans notre livre de raison au feuillet $126 \mathrm{v}^{\circ}$ ), petit-fils d'Étienne Bernard. Les lettres sont conservées à Langres, Bibliothèque du Grand Séminaire, MS 101 dans un recueil factice compilé par Théodecte Tabourot, son petit-fils. Notons que Théodecte Tabourot est né l'année de la mort d'Étienne Bernard. Ces lettres furent éditées et commentées par H. Drouot.

33. Étienne Bernard (1553-1609), avocat et magistrat, fut nommé lieutenant général du bailliage de Chalon-sur-Saône. Il fut pourvu de l’office de garde des sceaux grâce aux divisions amenées par la Ligue. 


\section{7}

\section{Le 27 de decembre de cette année mourut mon cousin maitre Bernard Coussin ${ }^{37}$ d'une melancholie ${ }^{38}$ dont je fus tresmarry.}

\section{8}

\section{Le 24 de janvier 1608 naquit Gabrielle ${ }^{39}$ à 4 heur[es] un quart apres midy et le 25 fut baptisée à Saint Michel $^{40}$. Son parrain fut monsieur Maclou}

Étienne Bernard a laissé la réputation d'un avocat très distingué. Député du Tiers de Bourgogne aux États généraux de Blois, il y joua un rôle fort important et fut choisi comme orateur du Tiers État de France. Il fut encore député du Tiers pour la Bourgogne, étant vicomte-mayeur de Dijon, aux États généraux de la Ligue tenus à Paris en 1593. Charles, duc de Mayenne, après lui avoir accordé la charge de conseiller garde des sceaux de la Chancellerie, nomma Étienne Bernard en 1594 président de la chambre de justice de Marseille. Après la Ligue, il fut lieutenant général au bailliage de Chalon en 1597. Étienne Bernard épousa Marguerite Paradin en 1575 dont il eut : Jean, l’âné, qui épousa Jeanne de Pontoux en 1600 ; Claude, surnommé «le pauvre prêtre " (voir note n 56) ; Jeanne, qui épousa Guillaume Tabourot, seigneur de Saint-Apollinaire ; Élisabeth, qui épousa Nicolas Mathieu, seigneur de Frontenard, lieutenant du bailliage de Chalon ; Pernette, qui semble avoir épousé Antoine Roux (voir note $n^{\circ}$ 62). Sur Étienne Bernard, voir Palliot, 256-258.

34. Marguerite Paradin, première femme d’Étienne Bernard, décède en 1597 : « [...] il a laissé à Dijon, dans son logis du temps de la Ligue, situé place Saint-Michel, plusieurs de ses enfants : Jeanne sa fille aînée, alors âgée de quinze ans et demi, Élisabeth, et peut-être la dernière-née Pernette [...] Cependant, c'est, on le verra, Jeanne, si jeune encore, qui doit ordonner la vie domestique... » (Drouot, 163). 35. « Monsieur Bernard mon beau frere » : marié depuis peu, Guillaume Bernard ne précise pas de quel membre de la famille Bernard il peut s'agir. Comme candidats, frères de sa nouvelle épouse Jeanne Bernard, il y a Jean Bernard, lieutenant général de Chalon (né en 1576) (voir note nº 54) ou encore le jeune Claude Bernard (1588-1641), qui deviendra le « pauvre prêtre ", mais après 1617.

36. Sur les voyages en Italie entrepris par Guillaume Tabourot, Étienne Bouhier, Jean (ou Claude ?) Bernard, voir Gulczynski, « La construction du château de Cormatin... », 38, note 57. Ce milieu était fortement imprégné d'italianisme, influencé par des séjours d'études ou d'amateurs esthètes. Ainsi, Étienne Bouhier (c. 1580-1635) a effectué des études à Padoue ; Guillaume Tabourot semble y avoir rejoint son ami en 1606. Ronsin, 22.

37. Bernard Coussin, mort en 1607, avocat, était échevin de Dijon de 1598 à 1599. Des vers latins de Bernard Coussin figurent dans le Dictionnaire des rimes françoises... de Jean Lefèvre ( $2^{\mathrm{e}}$ ed. revue par Étienne Tabourot ; voir ci-dessus note no 3 ) à la sig. i ii verso.

38. Melancholie ou variante merencolie, dont l'un des sens est « Mourir par merencolie», par consomption, ou d'une crise de bile noire secrétée par la rate. Voir Frédéric Godefroy, Dictionnaire de l'ancienne langue française et de tous ses dialectes $d u I X^{e}$ au XVe siècle, 10 vols. (Paris, 1880-1895), $5: 222$.

39. Gabrielle Tabourot, fille de Guillaume Tabourot, née en 1608, épousa en 1630 Prudent de Monginot, fils de Nicolas, avocat au Parlement de Paris. Voir la Notice historique sur la famille Désir de Fortunat 
Brocard $^{41}$ capitaine des gardes de monsieur nostre gouverneur et marraine damoiselle Gabrielle Chiquot de Monpastét ${ }^{42}$ ma mere.

Le 26 mars suivant mademoiselle Guillemette Tabourot, vefve de feu monsieur le conseiller Fremiot ${ }^{43}$ ma cousine, mourut.

\section{9}

Le lundy 23 de mars 1609, monsieur le lieutenant Bernard ${ }^{44}$ mourut à Chalon, plaint et regretté de tous les gens de bien.

Le 18 de may suivant à six heures du matin, pendant que j'estois à Chalon, naquit Theodecte Tabourot. Son parrain fut monsieur l'official ${ }^{45}$ mon oncle

et les familles alliées par C. Faure et Henri Désir de Fortunat, Mémoire de la Société d'histoire et d'archéologie de Châlon-sur-Saône 2.11 (1923) : 43. Le couple eut un fils prénommé Théodecte, prénom familial souvent donné par les Tabourot. Prudent de Monginot se convertit au protestantisme et épousa en secondes noces Jeanne Perny. La naissance de Gabrielle Tabourot, première fille du couple Guillaume Tabourot et Jeanne Bernard est aussi évoquée dans une lettre d'Étienne Bernard, datée du 24 janvier 1608 : «Monsieur Tabourot, je viens de recevoir la bonne nouvelle de la naissance de ma petite fille. Dieu soit loué de la santé de la mere. Elle prendra courage de nous faire un Estienne cy après et scais que vous ne dedirés pas...» (cité par Drouot, 170).

40. L'église Saint-Michel de Dijon est une construction du XVI'e siècle, consacrée en 1529 par Philibert de Beaujeu, évêque de Tonnerre.

41. Maclou Brocard fut nommé curateur des enfants mineurs d'Étienne Bernard, beau-père de Guillaume Tabourot. Il avait épousé Marguerite Noblet, cousine germaine d'Étienne Bernard.

42. Gabrielle Chiquot de Montpastey (morte en 1628), épouse d'Étienne Tabourot. Ils se marient fin 1572 ou début 1573. Cette même année, Étienne Tabourot sera déshérité par sa mère Bernarde Thierry (Choptrayanovitch, 37-38).

43. Guillemette Tabourot avait épousé André Frémiot, conseiller puis président du Parlement de Dijon. La famille Frémiot (ou Frémyot) comptait parmi les plus notables de Bourgogne. Sur les Frémiot, incontournable famille de magistrats bourguignons (voir Palliot, 86, 178, 217, 265). André Frémiot était l'oncle de Jeanne-Françoise Frémyot de Rabutin, baronne de Chantal, dite sainte Jeanne de Chantal, proche de saint François de Sales, qui fonda en 1610 l'Ordre de la Visitation, et fut l'arrière-grand-mère de Madame de Sévigné.

44. Étienne Bernard (1553-1609), beau-père de Guillaume II Tabourot, père de Jeanne Bernard. Citons l'ouvrage suivant: Harangue funèbre faite sur le trespas de Monsieur Bernard et prononcee en l'eglise des Peres Minimes à Chalon l'an mil six cent neuf par le R.P. Crochard, theologien minime en la province de Bourgongne, $2^{\mathrm{e}}$ éd. (Paris : J. Dedin, 1633).

45. Théodecte Tabourot (mort en 1631), second fils d'Étienne Tabourot, auteur des Bigarrures. Après avoir fait ses études de théologie, il fut reçu chanoine à Langres en 1583, où il devint chantre, puis official (c'est-à-dire l'ecclésiastique chargé de la juridiction contentieuse du chapitre). C'est donc le frère de 
par son procureur special monsieur le lieutenant Tabourot mon bon cousin ${ }^{46}$. Sa marraine mademoiselle Catharine Juret, femme de monsieur Bohyer ${ }^{47}$ conseiller au Parlement. Hunc placido lumine videant planetae ${ }^{48}$.

\section{0}

\section{Le vendredy 9 de juillet naquit sur les sept heures trois quarts du matin Judith $^{49}$ et fut baptisée le 13 à Nostre Dame ${ }^{50}$. Sa marraine fut damoiselle Judith}

Guillaume II Tabourot, auteur du présent livre de raison, et l'oncle de ses enfants. Théodecte Tabourot possédait une bibliothèque : citons par exemple un exemplaire du De diversa hominum natura... (Lyon, 1549), Lyon, Bibliothèque municipale, B 511371, avec un ex-libris et des notes de la main de Théodecte Tabourot (mort en 1631).

46. «Monsieur le lieutenant Tabourot mon bon cousin » : il y a un Alexandre Tabourot, qui bénéficiait en 1578 de la charge de lieutenant de la mairie de Dijon. Cette même charge avait été accordée en 1574 à Étienne Tabourot et Jean Fyot (voir Choptrayanovitch, 48). Cet Alexandre Tabourot, seigneur de Bricon, signe une pièce dans la seconde édition augmentée et reprise par E. Tabourot du Dictionnaire des rimes françoises... (1587) de Jean Lefèvre : «Sonnet au lecteur par Alex. Tabourot, sieur de Bricon » (sig. e. ii recto). Dans le cas présent, il doit s'agir plus vraisemblablement d'Alexandre Tabourot (mort en 1654), seigneur de Véronnes, lieutenant général à la table de marbre du Palais à Dijon (c'est-à-dire lieutenant à la juridiction des Eaux et Forêts). Ce sont des membres de la branche de Véronnes de la famille Tabourot, cousins de Guillaume Tabourot (branche des seigneurs de la Tour de Saint-Apollinaire). Guy Tabourot de Véronnes et Guillaume I Tabourot de Saint-Apollinaire avaient pour père commun Pierre $\mathrm{I}^{\text {er }}$ Tabourot de Véronnes, vicomte maïeur de Dijon (mort en 1552).

47. Catherine Juret, veuve Massol, avait épousé en secondes noces Jean II Bouhier (1546-1620), conseiller au Parlement de Dijon. De son premier mariage avec Catherine Millière, Jean II Bouhier avait eu un fils Étienne Bouhier, grand ami de Guillaume Tabourot. En 1604, Étienne Bouhier épousa en premières noces Claude Massol, la fille de sa belle-mère Claude Juret : Guillaume Tabourot est cité parmi les amis et alliés dans leur contrat de mariage (Dijon, Archives départementales de la Côte-d'Or, notaire Aubert Gelyot, $n^{\circ}$ 1716).

48. Seul passage en latin dans ce livre de raison. Hunc placido lumine videant planetae : "Que les planètes le voient sous une douce lumière ». Cette inclusion témoigne du degré de culture de Guillaume Tabourot. 49. Judith Tabourot rentra au couvent des Ursulines le 20 juillet 1625.

50. L'église Notre-Dame de Dijon s'élève à proximité du Palais des Ducs et fut construite au XIII ${ }^{e}$ siècle, avec sa façade caractéristique et unique dans l'architecture gothique française. Ce fut la première paroisse de Dijon, édifiée à la place de l'ancienne chapelle du marché. C'est dans cette église que les maires venaient prêter serment après leur installation. Voir Joseph Bresson, Histoire de l'Église NotreDame de Dijon depuis ses origines jusqu'à la fin du XVIII siècle (Dijon : Union typographique, 1891). Presque tous les enfants du couple Tabourot-Bernard qui suivirent furent baptisés à Notre-Dame de Dijon. À noter, Étienne Tabourot, littérateur, fut enterré à Saint-Bénigne de Dijon. 
Quenot ${ }^{51}$ vefve de feu monsieur le lieutenant Bernard et son parrain monsieur Rougeot $^{52}$ mon beau frere.

\section{1}

Le dimanche 9 d'octobre jour de Saint Denys naquit a une heure un quart apres midy Jaqueline et fut baptisée à Nostre Dame. Sa marraine fut damoiselle Jaquette Bouvot ${ }^{53}$, nostre cousine, et eut pour parrain monsieur le lieutenant Bernard $^{54}$, mon beau frere.

\section{3}

Le lundy 25 de febvrier lendemain de Saint Mathias naquit Charlotte à deux heures environ quinze minutes apres midy. Sa marraine fut dame Charlotte Bourgeois, femme de monsieur le président Desbarres ${ }^{55}$. Et son parrain maitre Claude Bernard ${ }^{56}$, advocat à la Cour, son oncle maternel.

52. Il peut s'agir du beau-frère de Guillaume Tabourot, issu de sa première alliance avec Louise Rougette, avant son second mariage avec Jeanne Bernard en 1606.

53. Jacquette Bouvot, membre de la famille Bouvot, dont Claude Bouvot était pourvu d'un office de maître ordinaire à la Chambre des comptes de Dijon (voir d'Arbaumont, 179-180).

54. Jean Bernard, lieutenant-général de Chalon, né en 1576, successeur de son père Étienne Bernard. Jean Bernard épouse à Chalon Jeanne de Pontoux en 1600.

55. Pierre Desbarres fut conseiller au Parlement, puis président en 1611. Il signe comme témoin le contrat de mariage de Guillaume Tabourot en 1606. Pierre Desbarres, seigneur de Ruffey et Echirey, conseiller d'État et président au parlement en 1611, avait épousé en 1599, Charlotte, fille de Claude Bourgeois, seigneur de Moleron et conseiller au parlement.

56. Il s'agit de Claude Bernard (1588-1641), frère de Jeanne Bernard, effectivement beau-frère de Guillaume II Tabourot. Dans notre livre de raison, il est dit « avocat » en 1613. De fait, en 1613, Claude Bernard n'est pas encore entré dans l'état ecclésiastique, sous l'influence du prélat Jean-Pierre Camus, évêque de Belley venu à Dijon en 1615. Ce sera Jean-Pierre Camus qui mettra Claude Bernard en relation avec saint François de Sales (voir De Broqua, 29-33). Claude Bernard deviendra après 1617 celui que l'on désignait sous l'appellation de " Pauvre prêtre ", ecclésiastique réputé pour sa charité. Sur les rapports tendus de Claude Bernard avec son père Étienne Bernard et sa vie mouvementée avant ses vœux pieux, voir Drouot, 164-165 ; sur Claude Bernard dit le "pauvre prêtre ", on consultera l'ouvrage précité par Joseph Jean de Broqua. Après sa conversion, il consacra toute sa fortune, qui était considérable, aux œuvres de charité et refusa tous les honneurs qu’on voulut lui conférer. 


\section{4}

Le mardy 13 de may 1614 a la demie apres minuit naquit Oudette [fol. $127 \mathrm{r}^{\circ}$ ] Tabourot baptisée le lendemain à Nostre Dame. Sa marraine fut damoiselle Oudette Maillard, femme de monsieur le thresorier Legouz ${ }^{57}$, mon cousin, et eut pour parrain monsieur le lieutenant Tabourot ${ }^{58}$.

\section{5}

Le lundy 23 febvrier veille de Saint Mathias mourut damoiselle Didiere Tabourot femme du fut ${ }^{59}$ sieur Coussin ${ }^{60}$ père qui avoit eu tout le bien de nostre maison. Le lundy 23 novembre, jour de Saint Clement, naquit Estienne Tabourot à neuf heures trois quarts du matin, baptisé le lendemain à Nostre Dame. Son parrain fut monsieur le secretaire Arviset ${ }^{61}$ et sa marraine damoiselle Perrenette Bernard $^{62}$, femme de monsieur le maistre des comptes Roux.

57. Odette Maillard avait épousé en 1603 Pierre Legoux, écuyer et trésorier de France en la généralité de Bourgogne et de Bresse, seigneur de Vallepelle ou Vellepesle (voir Jules Thomas, Épigraphie de l'Église Notre-Dame de Dijon [Dijon et Paris : Nourry éditeur, 1904], 19-20). Odette Maillard est morte en 1640. 58. Voir note $\mathrm{n}^{\circ} 46$ ci-dessus.

59. «Fut » - feu, défunt. Voir Edmond Huguet, Dictionnaire de la langue française du XVI siècle, vol. IV (Paris : E. Champion, 1925-1973), 245.

60. Didière Tabourot épousa Bernard Coussin. La remarque « qui avoit eu tout le bien de nostre maison » doit faire référence à l'héritage de Guillaume Tabourot et Bernarde Thierry, parents de Didière Tabourot, mais aussi d’Étienne Tabourot, littérateur mort en 1590. En contractant un mariage avec Gabrielle Chiquot de Monpastey, Étienne Tabourot s'est vu déshérité par sa mère Bernarde Thierry. L'héritage passa donc à la sœur d'Étienne Tabourot, à savoir Didière Tabourot (Théodecte, leur frère étant chanoine à Langres).

61. Émilian Arviset était conseillier secrétaire du roi en la chancellerie de Bourgogne. Seigneur de la Cosme, Colonge et de Marcilly lès Mont Saint-Jean, fut reçu conseiller laïc au Parlement en 1606 (Palliot, 273).

62. Pernette Bernard doit être la sœur de Jeanne Bernard, toutes deux filles d'Étienne Bernard. Elle est citée parmi les filles d'Étienne Bernard, voir Drouot, 163 : « [...] et peut-être la dernière-née, Pernette... ». Maître Roux doit être Antoine Roux, maitre ordinaire à la Chambre des comptes de Dijon, reçu en 1616 (voir d'Arbaumont, 210). 


\section{7}

Le mardy jour de Toussaints 1 de novembre naquit Pierre Tabourot à quatre heures un quart apres midy et fut baptisé le jeudy suivant à Nostre Dame. Il eut pour parrain monsieur le conseiller Saumaize dit de Chazans ${ }^{63}$ et pour marraine damoiselle Magdalene Giroux, femme de monsieur le conseiller Bouhyer ${ }^{64}$ fils, mon bon amy.

\section{9}

Le jour de Saint Marc 25 d'apvril, un jeudy à deux heures et demie apres midy, naquit Nicolas et fut baptisé le 2 de may suivant. Son parrain fut monsieur le lieutenant particulier Mathieu ${ }^{65}$, de Chalon pour lequel monsieur Roux ${ }^{66}$ presta la main et sa marraine fut mademoiselle Claude Joly, femme de monsieur le thresorier Gaigne ${ }^{67}$.

63. Il doit s'agir de Pierre Saumaize ou Saumaise, seigneur de Chazans, de Nanteuil, de la Tour, Villars etc., conseiller « laïc » au parlement, car son père Jérôme Saumaize, seigneur de Chazans mourut en 1614 (Palliot, 282-283). Alexandre Tabourot, neveu de Guillemette Tabourot (voir note no 46), avait vendu un hôtel connu sous le nom de Hôtel Saumaise de Chasans à Dijon.

64. Étienne Bouhier (c. 1580-1635), « mon bon amy », était le fils de Jean II Bouhier et de Catherine Millière. Il épousa en 1604 Claude Massol, la fille de la deuxième femme de Jean II Bouhier (son père), puis en secondes noces Madeleine Giroux. Il fut nommé conseiller au Parlement de Dijon en 1607 et poursuivit la constitution de l'importante bibliothèque familiale (voir l'étude d'Albert Ronsin déjà citée). À titre d'exemple de la culture savante et littéraire d'Étienne Bouhier, citons au hasard un manuscrit de Chrétien de Troyes, lui venant de son père (Montpellier, BI, Sect. Med. H 249) ; voir Keith Busby et al., éd., The Manuscripts of Chrétien de Troyes (Amsterdam-Atlanta : Rodopi, 1993), 100. Ronsin parle de la «bibliothèque de neuf générations " pour caractériser la bibliothèque des Bouhier à Dijon (Ronsin, 20). 65. Nicolas Mathieu, époux d'Élisabeth Bernard (elle-même sœur de Jeanne Bernard), était lieutenant particulier du bailliage de Chalon (voir Drouot, 165, note 1). On lit dans le testament de Claude Bernard : "Item, donne \& legue à Madame Mathieu sa sœur, femme de Monsieur le lieutenant particulier de Châlons sur Saosne, trois autres petits tableaux garnis d'argent " (Thomas Le Gauffre, La vie de C. Bernard dit le Pauvre Prestre [Paris : De la Caille, 1680], 382).

66. Voir note $\mathrm{n}^{\circ} 62$ ci-dessus.

67. Nicolas Gagne (1580-?) épouse Claude Joly, fille d'Antoine Joly, baron de Blaisy et greffier en chef au parlement dijonnais (1612). Il devient ensuite trésorier général de la généralité de Bourgogne. Enrichi, il achète en 1650 Perrigny, une petite seigneurie proche de Dijon. 
1620

Le lundy 16 de novembre naquit Jeanneton à trois heures et demie avant midy. Son parrain fut monsieur le conseiller Massol ${ }^{68}$ mon cousin et sa marraine dame Jeanne Vadot, femme de monsieur le president Giroux ${ }^{69}$, et fut baptisé le mardy à Nostre Dame.

\section{2}

Le mardy 23 mars naquit à deux heures et demie du matin Marc Anthoine Tabourot, battisé le meme jour à Nostre Dame. Il eut pour parrain monsieur Marc Anthoine de la Tour ${ }^{70}$, mon cousin, et pour marraine damoiselle Marthe Barbisey ${ }^{71}$, fille de monsieur le president. Il mourut le 30 suivant et est enterré à Nostre Dame en la chapelle de Bergeon ${ }^{72}$.

68. Jean Massol, seigneur de Savigny-sous-Beaune, conseiller « laïc » au parlement de Bourgogne en 1603 (Palliot, 170).

69. Philippe Giroux (exécuté en 1643) était président à mortier au Parlement de Bourgogne et ancien favori du Prince de Condé. Signalé par son cousin Jean Baillet, Président de la cour des comptes de Bourgogne, au gouverneur de la province, comme l'auteur probable de placards anonymes à l'encontre du Prince de Condé, Philippe Giroux en garde un énorme ressentiment à l'égard du Président Baillet. À cela s'ajoute un autre motif, bien moins avouable : après s'être débarrassé de sa première femme en l'empoisonnant, le président Giroux souhaite épouser la femme de Baillet, avec laquelle il entretenait depuis longtemps des relations licencieuses. Tout se joua lorsque le Prince de Condé commit l'imprudence de vouloir réconcilier les deux cousins. Le président Baillet se rend le 6 septembre 1638, accompagné de son valet de chambre, à l'hôtel Giroux, où tous deux furent assassinés par Giroux et ses complices. Leurs corps sont alors jetés dans les latrines. Le procès qui s'ensuivit se termina par un arrêt de mort à l'encontre du président Giroux et eut un grand retentissement en Bourgogne, où l'assassin et la victime étaient tous deux liés aux familles les plus riches et les plus puissantes de cette province. On conserve un récit complet et détaillé de cette affaire dans Élisabeth-François Lacuisine, Le Parlement de Bourgogne depuis son origine jusqu'à sa chute, 3 vols. (Dijon : Rabutot, 1864), vol. II. Plus récemment, James R. Farr a analysé plus en profondeur cette affaire : voir James R. Farr, « The Death of a Judge: Performance, Honor, and Legitimacy in Seventeenth-Century France », Journal of Modern History 75.1 (2003) : 1-22 ; James R. Farr, A Tale of Two Murders : Passion and Power in Seventeenth-century France (Durham, Londres : Duke University Press, 2005).

70. Marc Antoine de la Tour, cousin éloigné de Guillaume II Tabourot : Didière Tabourot (fille de Guy Tabourot (seigneur de Véronnes), oncle d'Étienne Tabourot, le littérateur) avait épousé Claude de la Tour, seigneur de Villiers-le-Duc.

71. Marthe Berbisey, fille de Claude Berbisey, seigneur de la Tour-de-Pouilly, de Sainte-Marie-la-Blanche et en partie de Vonges, Tart-le-Bas et Varanges, qui acheta en 1555 une charge de maître des comptes. Marthe Berbisey épousa Claude Frémiot, président aux comptes.

72. Il existait à Notre-Dame de Dijon une chapelle dite de Barjon au-dessus de laquelle il y avait une chambre où les fabriciens tenaient leurs assemblées (Bresson, 327). 
1628

Le 21 decembre damoiselle Gabrielle Chiquot de Monpastey ma mere mourut et fut enterrée à Saint Jean ${ }^{73}$.

[fol. $127 \mathrm{v}^{\circ}$ ]

\section{9}

Le [blanc] de janvier 1629, le roy Loys 13 de ce nom fit son entrée en cette ville, allant en Piedmont ${ }^{74}$.

\section{0}

Le dimanche 23 de juin Charlotte fut receue religieuse au couvent de Saint Claire à Bellegarde ${ }^{75}$.

73. Il s'agit du cimetière de Saint-Jean à Dijon.

74. Allant en Piedmont : En pleine guerre de Trente Ans se déroulait en périphérie la guerre de la succession de Mantoue, opposant la France au Saint Empire romain germanique. Vincent II Gonzague, duc de Mantoue et de Montferrat, avait marié sa nièce Marie à Charles III, duc de Nevers. Ferdinand II, saint empereur romain germanique, ne reconnut pas l'autorité de Charles III, duc de Nevers, seigneur français sur le duché de Mantoue. Ferdinand II s'allia avec Charles Emmanuel I ${ }^{\mathrm{er}}$, duc de Savoie contre le roi de France Louis XIII. En 1628, Charles Emmanuel I ${ }^{\text {er }}$ lança les hostilités. Accompagné par des troupes espagnoles, il s'empara de Montferrat, contraignant Charles de Nevers à reculer. En début d'année 1629, Louis XIII se retrouvait dans une situation délicate : il laissait faire le duc de Savoie, afin de préserver l'alliance espagnole, ou il intervenait et s'attaquait ouvertement à l'Espagne. La reinemère était favorable à la première théorie, Richelieu, à la seconde. Le roi de France, soucieux de ne pas laisser les coudées franches à l'Espagne, décida d'intervenir en Italie. Traversant les Alpes à la tête de l'armée du siège de La Rochelle (mars 1629), le roi de France vint au secours de Charles de Nevers. L'armée royale française poursuivit les Savoyards jusqu'à Suze et Charles Emmanuel I ${ }^{\mathrm{er}}$, conscient de son infériorité, fut alors contraint de négocier. Signant le traité de Suze (avril 1629), Charles Emmanuel Ir de Savoie reconnut Charles de Nevers comme légitime duc de Mantoue et Montferrat. En juin 1630, après avoir envahi la Savoie, Louis XIII décida de marcher contre le Piémont. En juillet, l'armée royale s'empara de Saluces, et Charles Emmanuel Ir de Savoie mourut peu de temps après.

75. Ce n'est pas un hasard qu'une des filles de Guillaume Tabourot et Jeanne Bernard soit admise au couvent de Sainte-Claire de Bellegarde (Seurre, dép. Côte-d’Or), au vu des fonctions de Guillaume Tabourot comme bailli de Bellegarde depuis 1619. La ville de Seurre prit le nom de Bellegarde jusqu'en 1650. Située dans la vallée de la Saône, au confluent de deux bras de la rivière navigable, la petite ville est un lieu de passage de la rivière au sud du département de la Côte-d'Or, à $40 \mathrm{~km}$ de Dijon. Les Cordelières ou dames de Sainte-Claire de Seurre s'établirent à Seurre (anciennement Bellegarde) en 1421, fondées par sainte Colette. Voir Paul Guillemot, Histoire de Seurre suivie de ses chartes d'affranchissement (Beaune : Batault-Morot, 1859), 39. 


\section{Le lundy 29 juillet, Gabrielle fut mariée au Sieur Monginot ${ }^{76}$, advocat demeurant à Lengres et furent faictes ces nopces à Saint Aplomet ${ }^{77}$.}

\section{1}

Le mardy 21 de janvier Theodecte Tabourot ${ }^{78}$ fut receu chanoine en l'eglise de Saint Mammets de Lengres ${ }^{79}$ par la demission de monsieur l'official Tabourot mon oncle. Ce ne fut pas sans peine.

Le vendredy 7 febvrier suivant de la mesme année 1631, ledit sieur Tabourot ${ }^{80}$ mon oncle mourut en sa maison à Lengres et me laissa son heritier suivant la coustume de Sens ${ }^{81}$.

76. Gabrielle Tabourot épousa Prudent Monginot, voir note no 39 ci-dessus.

77. Saint-Aplomet ou Saint-Applume sont les formes anciennes pour la commune de Saint-Apollinaire (Côte-d'Or), sise en périphérie de Dijon, dont Guillaume Tabourot était le seigneur. On citera le dessin connu d'Étienne Martellange, légendé Saint-Applume a Mr. Tabourot 19 septembre 1610. Veüe de SaintApplume (Paris, BnF, Dép. Estampes et photographies, Réserve UB-9-Boîte FT 4).

78. Théodecte Tabourot, chanoine du chapitre cathédrale de Langres, petit-fils d'Étienne Bernard, fils de Guillaume Tabourot et de Jeanne Bernard.

79. L'église Saint-Mammès de Langres : il s'agit de la cathédrale Saint-Mammès de Langres (HauteMarne), édifiée au XII ${ }^{e}$ siècle au centre du quartier canonial. Sur les chanoines, voir Georges Viard, "Les chanoines de Langres au XVII ${ }^{e}$ siècle : recrutement, origines, fortunes », Annales de l'Est 5.XXVIII (1976) : 87-138.

80. Théodecte Tabourot, frère d'Étienne Tabourot (mort en 1590), était l'oncle de Guillaume II Tabourot : il était chanoine de Langres. C'était donc le grand-oncle de Théodecte (le jeune) Tabourot (fils de Guillaume II Tabourot et Jeanne Bernard) dont il prend la charge de chanoine en remplacement. Théodecte (l'ancien) Tabourot avait des livres dont on citera celui décrit par Léon Techener, Bibliothèque champenoise (Paris : 1886, Genève : Slatkine Reprints, 1972), n 337 : « Sur le titre du Juvénal, on lit : Sum Theodecti Taborti canonici lingonensis; et au-dessus on a dessiné à la plume, un tambour, armes parlantes des Tabourot... ». Notons que plusieurs Tabourot se sont succédé comme chanoines à Langres, puisque Théodecte (l'ancien) remplaçait déjà Jean Tabourot, chanoine et théologal de Langres mort en 1595.

81. La coutume de Sens désigne l'ensemble des règles de droit coutumier qui régissaient les rapports juridiques, économiques, familiaux et sociaux des habitants de la ville de Sens, sise dans le duché de Bourgogne. Effectivement, selon la coutume de Sens, les biens d'un chanoine, tel Théodecte Tabourot, ne revenaient pas de fait à l'ordre des chanoines de Langres : " Les biens d'une personne qui entre en religion apres la profession n'appartiennent au Monastère ou Religion en laquelle est faicte ladicte profession : mais viennent à ses plus prochains parens... " (Coustumes du bailliage de Sens et anciens ressorts d'iceluy [Sens, 1622], 60). 


\section{XXXXIIII $[1644]^{82}$}

Le sammedict dixzeme de septembre, mon cher mariy moureu [entres onze heure et] à la menuis soude[ne]mant. Dieu par sa bonté luy face misericorde. Yl et estés plaint et regretté de tout les gens de bien. Yl et entarré au reverant père iacoupin ${ }^{83}$ au y sept demandée ${ }^{84}$.

Mon filz Estienne Tabourot et sa famme m'on faict ce bien et ce plaisir de m'an voiée [sic] leur peteite fille ma filloulle ${ }^{85}$ Jeanne Tabourot ${ }^{86}$ le jour de la sainte Trenité 16 XXXXV [1645]. Elle feut batisé le xxii [22] jeun 16XXXXV [1645]. Dieu la face bien saige.

82. Changement de main. Il s'agit de la main de Jeanne Bernard, femme (et désormais veuve) de Guillaume II Tabourot. À noter que Jeanne Bernard — fille d'Étienne Bernard, important personnage public - apparaît comme semi-lettrée, avec une orthographe qui conserve des traces d'oralité et une mauvaise maitrise de la grammaire.

83. Au reverant père iacoupin : il s'agit de l'église des Dominicains ou Jacobins, à Dijon. L'entourage du duc Hugues III installe un couvent en 1237, établissement urbain dès son origine au cœur de Dijon, appelé Ave Maria. Le couvent de l'Ave Maria devient vite un centre intellectuel réputé à Dijon.

84. Sens de ce passage : où il avait demandé (à être enterré) ?

85. «Filloule» : variante de « filleule». Forme attestée dans Huguet, vol. IV, 106.

86. Jeanne Tabourot, fille d'Étienne Tabourot (né en 1615). 